\title{
ON THE STRUCTURE OF PREMIXED AND DIFFUSION LAMINAR SPHERICO-SYMMETRICAL FLAMES
}

\author{
P. PÉREZ DEL NOTARIO AND C. SĀNCHEZ TARIFA
}

\begin{abstract}
Premixed and diffusion laminar spherico-symmetrical flames are studied by means of a non-adiabatic model which considers variable temperature at infinity. The flame is supposed to be maintained by a porous sphere through which the gaseous mixture or one of the reactant gases is injected.

Stationary conditions are assumed and chemical kinetics of the process is approximated by means of an overall reaction rate.

Solution of the problem is achieved by means of an approximate analytical method. Flame temperature, mass flow per unit area at the flame surface, thickness and radius of the flame and temperature at the sphere surface are given as functions of the mass flow. The influence of the main parameters of the process: temperature at infinity, dimensionless ratio of the activation energy to the heat of reaction and sphere radius is also considered.

Some experimental results for diffusion flames are also given in the paper.
\end{abstract}

\section{Introduction}

The study of flames by means of sphericosymmetrical models presents several advantages in comparison with the studies performed by using one-dimensional models, especially for nonadiabatic cases.

Nonadiabatic conditions are natural in spherical flames owing to the heat transfer from the flame towards its surrounding atmosphere. On the contrary, nonadiabatic cases have to be introduced rather artificially in one-dimensional flames. Furthermore, truly one-dimensional flames are difficult to observe because of the lateral heat losses which cannot be avoided and because it is very difficult to produce a fluid motion absolutely perpendicular to the flame front.

A large number of studies have been performed on spherical flames in connection with droplet combustion. However, most of them referred to the diffusion flame of a fuel droplet burning in air, and such flames were studied disregarding chemical kinetics by assuming an infinitely fast reaction rate. There are a few studies on the decomposition spherical flame of monopropellant droplets $^{1,2}$ and on the combustion of fuel droplets considering finite chemical kinetics., ${ }^{3,4}$

On the contrary, there are very few studies on the theory of laminar spherical flames of the premixed type in which only gaseous species are involved in the process, and there are no studies, as far as we know, on diffusion spherical flames.

Spalding ${ }^{5}$ has studied a premixed spherical flame in which the gases emerge from a point source. The study is restricted to the adiabatic case and the reaction rate is considered to be only a function of the temperature. Several analytical and analog solutions are given. ${ }^{6,7}$

Westenberg and Favin ${ }^{8}$ have studied a premixed spherical laminar flame supported by a porous sphere. They studied only the adiabatic case and for a particular set of values of the principal parameters of the process.

The adiabatic case, in which the reaction products are supposed to extend indefinitely at the flame temperature, is the simplest one. Its interest lies in the possibility of comparing the results obtained with those of one-dimensional flames, but it seems very difficult to make an experimental model in which such conditions could be approximately reproduced

At the Instituto Nacional de Técnica Aeronáutica, Madrid, Spain, a research program on spherical flames is being conducted and a résumé of the results so far obtained are given in this paper.

Such results have been published in full in ref. 9. The theoretical program comprises the general study of both premixed and diffusion flames, and the theoretical model of the process has been selected in such a form that their conditions can be experimentally reproduced. The mixture or one of the gases is assumed to emerge from a porous sphere, which prevents chemical reaction within it due to a quenching effect. The study includes the analysis of the influence of the main parameters of the process such as temperature at infinity, activation energy, heat of reaction, sphere diameter, etc.

The experimental program comprises the obser- 
vation of diffusion and premixed butane-air and hydrogen-oxygen-nitrogen flames. Results for diffusion flames are given in the paper, as well as a qualitative comparison between theoretical and experimental results.

\section{Fundamental Assumptions}

The model of the process will be based on the following assumptions:

1. The flame is supposed to be maintained by a porous sphere through which the mixture or one of the gases is injected. It is assumed that chemical reaction does not take place within the sphere, and that chemical reaction goes to completion at infinity, where the temperature and composition of the atmosphere are given.

2. Stationary conditions will be considered, pressure will be taken constant and the process will be assumed to have spherical symmetry. Therefore, free convection effects are disregarded.

3. Only reactant species and reaction products will be considered. The influence of radicals is only exerted through the chemical kinetics of the process. This chemical kinetics will be approximated by means of an over-all reaction rate of any form, which may be deduced from the actual chemical kinetics of the process. ${ }^{1,3}$

4. The heat exchange through radiation between the hot gases and the porous sphere will be disregarded. For nonluminous flames this heat exchange is not important, and for luminous flames such exchange of radiant heat is small as compared to the heat transmitted through conduction, if the flame is close to the sphere surface.

On the other hand, the radiant heat exchange between the sphere at $T_{s}$ and the surroundings at $T_{\infty}$ will be taken into account.

5. Gases and their mixtures are assumed to be perfect gases. Mean values for the specific heats and thermal conductivities will be taken.

\section{General Equations and Boundary Conditions}

According to the foregoing assumptions the fundamental equations of the process and boundary conditions are as follows:

1. Continuity.

$$
\frac{\dot{m}}{4 \pi r^{2}} \frac{d \epsilon_{i}}{d r}=w_{i}
$$

2. Energy

$$
\dot{m}\left[-\left(\epsilon_{3}-\epsilon_{3 s}\right) q_{r}+\bar{c}_{p}\left(T-T_{0}\right)+\frac{4 \pi r_{s}^{2}}{\dot{m}} \sigma\left(T_{s}^{4}-T_{\infty}^{4}\right) e_{s}\right]-4 \pi r^{2} \bar{\lambda} \frac{d T}{d r}=0
$$

3. Diffusion.

$$
\sum_{j} \frac{Y_{j}}{M_{j}}\left[\frac{\dot{m} \bar{c}_{p}}{4 \pi r^{2} \bar{\lambda}}\left(\frac{\epsilon_{j}}{Y_{j}}-\frac{\epsilon_{i}}{Y_{i}}\right) \mathfrak{l}_{i j}-\frac{1}{Y_{i}} \frac{d Y_{i}}{d r}+\frac{1}{Y_{j}} \frac{d Y_{j}}{d r}\right]=0
$$

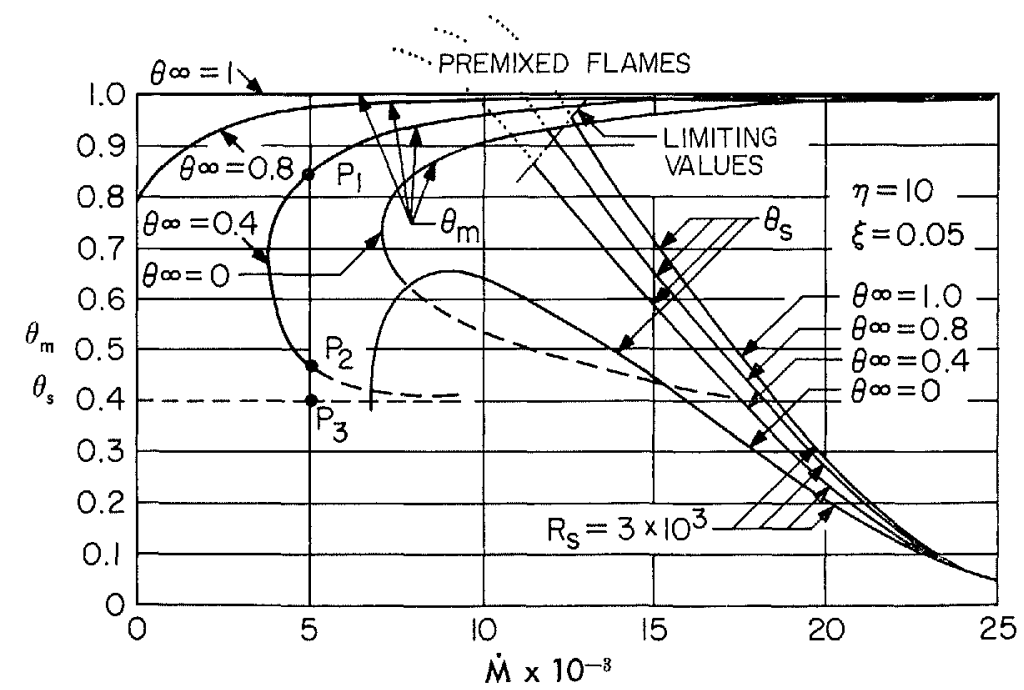

Fig. 1. Flame temperatures and temperatures at the sphere surface for premixed flames. 


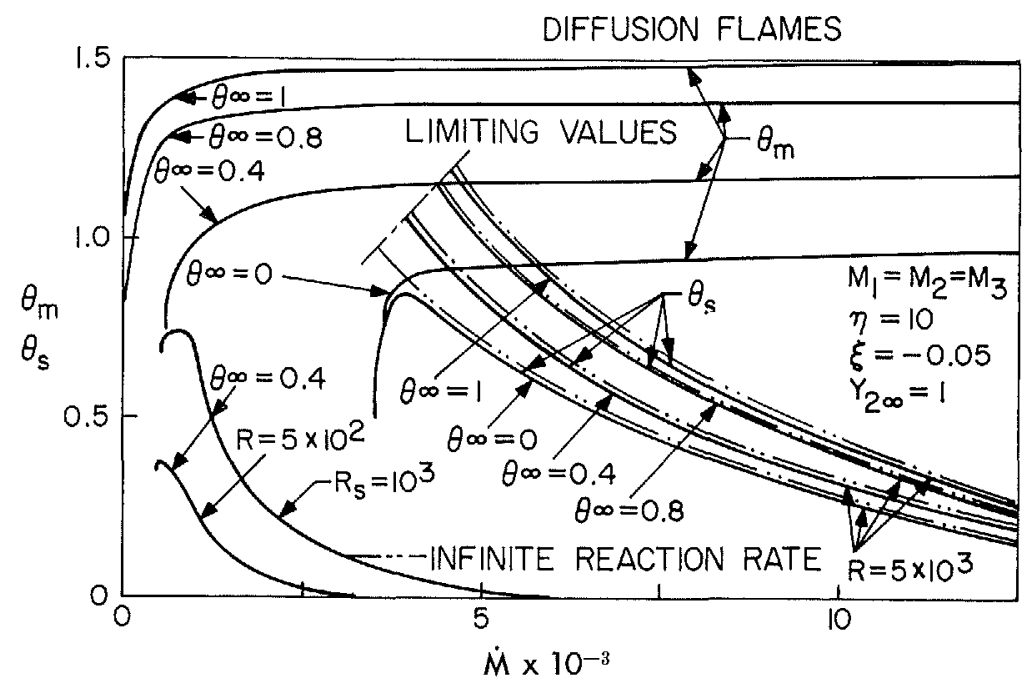

Fig. 2. Flame temperatures and temperatures at the sphere surface for diffusion flames.

4. Boundary conditions.

$$
\begin{aligned}
& r=r_{s}\left\{\begin{array}{l}
\epsilon_{1 s}=1 \\
\epsilon_{2 s}=\epsilon_{3 s}=0
\end{array}\right. \\
& r=\infty\left\{\begin{array}{l}
T=T_{\infty} \\
Y_{1_{\infty}}=0 \rightarrow \epsilon_{1_{\infty}}=0 \\
Y_{2}=Y_{2_{\infty}}=1-Y_{3_{\infty}}
\end{array}\right.
\end{aligned}
$$

Solution of the system gives functions $\epsilon_{i}(r)$, $Y_{i}(r)$ and $T(r)$ for given values of the mass flow $\dot{m}$ and sphere radius $r_{s}$. An expression of the reaction rate $w_{i}$ as a function of mass fractions and temperature should also be given.

Solution of the system is accomplished by means of an approximated analytical method which is developed in ref. 9.

\section{Theoretical Results}

Results are fully discussed in ref. 9. They have been obtained by taking a first order overall reaction rate for premixed flames and a second order over-all reaction rate for diffusion flames. In the present work only some of the more significant results, especially in connection with experimental values, will be discussed.

Dimensionless maximum flame temperature $\theta_{m}$ and dimensionless temperature* at the sphere surface $\theta_{s}$ are represented in Figs. 1 and 2 for

* Temperatures $\theta_{m}$ and $\theta_{s}$ are practically proportional to actual temperatures $T_{m}$ and $T_{s}$, because parameter $\xi$ is usually very small (See Nomenclature and ref. 9). premixed and diffusion flames as functions of the dimensionless mass flow $M$ for several values of the temperature at infinity $\theta_{\infty}$. It may be seen that flame temperatures depend considerably on mass flow and on $\theta_{\infty}$ at low values of $M$ for both types of flames, but they do not depend on the sphere size.

For premixed flames, when $\dot{M}$ is large all curves tend towards $\theta_{m}=1$, which is the temperature corresponding to the adiabatic case. On the other hand, for diffusion flames at large values of $\dot{M}$ all results tend towards those obtained by taking an infinitely fast reaction rate. This is the Burke and Schumann hypothesis for studying diffusion flames disregarding chemical kinetics, and in ref. 9 it is shown that such a hypothesis gives the asymptotic values of those obtained considering finite chemical kinetics.

When the temperature at infinity is small and in the region of small values of the mass flow three values of $\theta_{m}$ appear for each value of $M$ in both types of flames. These values correspond to three mathematical solutions of the problem, such as $P_{1}, P_{2}, P_{3}$ (Fig. 1). The actual physical existence of solutions $P_{2}$ and $P_{3}$, which correspond to combustion processes taking place at low temperature through a wide reaction zone, ${ }^{9}$ seems doubtful. Therefore, the results suggest that for nonadiabatic cases and when $\theta_{\infty}$ is small there exist minimum values of the mass flow under which combustion is not possible. This result is independent of the size of the sphere and the minimum value would also exist although the flame were originated from a point source.

Temperatures $\theta_{s}$ at the sphere surface are also shown for both types of flames in Figs. 1 and 2 


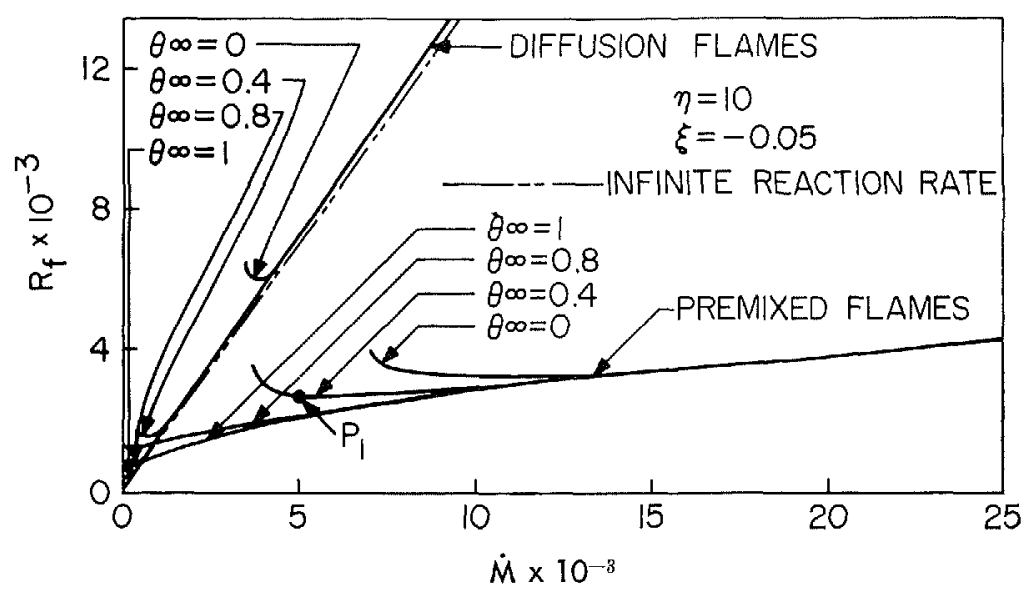

FIG. 3. Flame radius for premixed and diffusion flames.

as functions of the mass flow $\dot{M}$. These temperatures also depend on the sphere radius as shown in Fig. 2.

All curves $\theta_{s}=f(\dot{M})$ have a maximum value but in some curves the maximum does not appear because results are limited by the condition that the reaction zone must be located outside of the flame. Temperature $\theta_{s}$ increases rapidly until it reaches its maximum value and then it decreases slowly, tending towards zero.

Results for flame location are shown in Fig. 3. It may be seen that premixed flames are considerably closer to the sphere surface than diffusion flames.

Flame radius is practically independent of the sphere size, and its value decreases as the mass flow decreases. Therefore, for a given sphere radius there exists a minimum value of the mass flow under which the flame cannot be maintained, since the reaction zone should be located within the sphere. This minimum value is not related to the minimum value of the mass flow which was deduced from chemical kinetics considerations and it may have larger or smaller values depending on the sphere size and on the flame characteristics.

\section{Experimental Results}

An experimental investigation on diffusion and premixed spherical flames recently has been initiated. Diffusion flames have been studied by burning in air, butane gas, and hydrogen injected through different sized ceramic spheres.

Flames were not spherical due to free convection, except for very small values of the mass flow. Flame diameter was taken as the flame width measured at the mean horizontal plane of the sphere.
Flame temperatures and temperatures at the sphere surface were measured by means of small Pt-Pt-Rh thermocouples. These temperatures were corrected for radiation errors.

Flame temperature was taken as the maximum temperature at the aforcmentioned mean horizontal plane. This maximum value was obtained by displacing horizontally the thermocouple until a maximum temperature was reached. Temperatures at the sphere surface were measured at the same plane, inserting a half of the thermocouple bead within the sphere. These temperatures at the sphere surface were almost constant at different points on the lower hemisphere, but they were smaller at the upper hemisphere.

Experimental results are shown in Figs. 4 and 5. A good qualitative agreement between theoretical and experimental results could not be expected due to the disturbing influence of free convection. * However, the laws of variation of flame temperature and temperature at the sphere surface as functions of the mass flow are in excellent agreement with those predicted theoretically (curves $\theta_{m}=f(\dot{M})$ and $\theta_{s}=f(\dot{M})$ for $\theta_{\infty}=0$ of Fig. 2). The influence of the sphere size on $\theta_{s}$ also agrees very well with the theoretical results (Figs. 2, 4, and 5)

In the region close to the minimum values of the mass flow the flame temperature was a function of the sphere radius. In such a region the flame is located very close to the sphere surface, and combustion might be altered by the proximity of the sphere, since part of the reaction zone should be located within the sphere. This effect might explain the discrepancy between theoretical

* In order to avoid free convection effects, combustion of air and oxygen within hydrogen ${ }^{2}$ will be studied. 


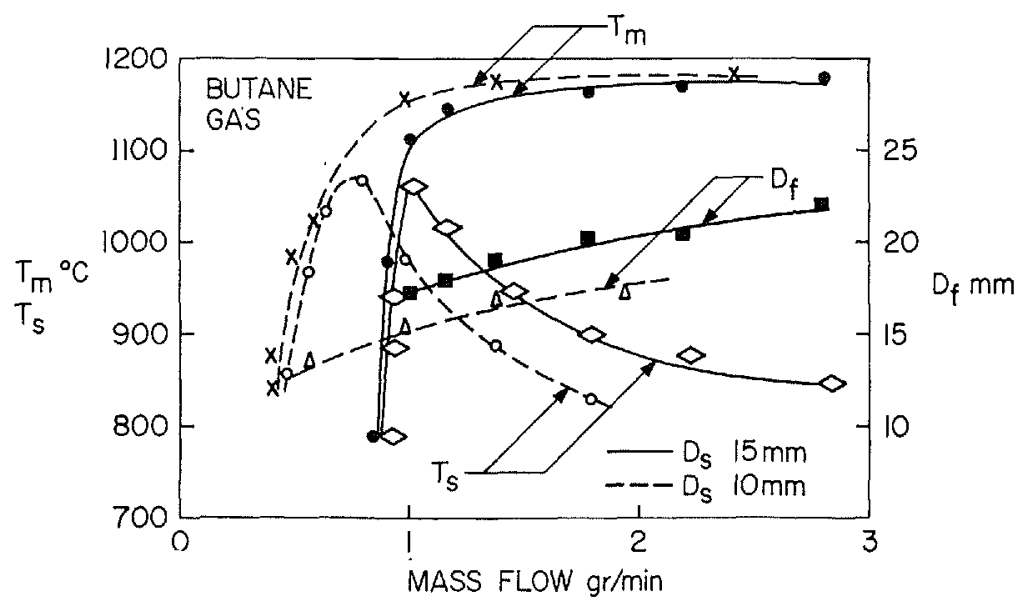

FIG. 4. Butane gas-air diffusion flame. Experimental results.

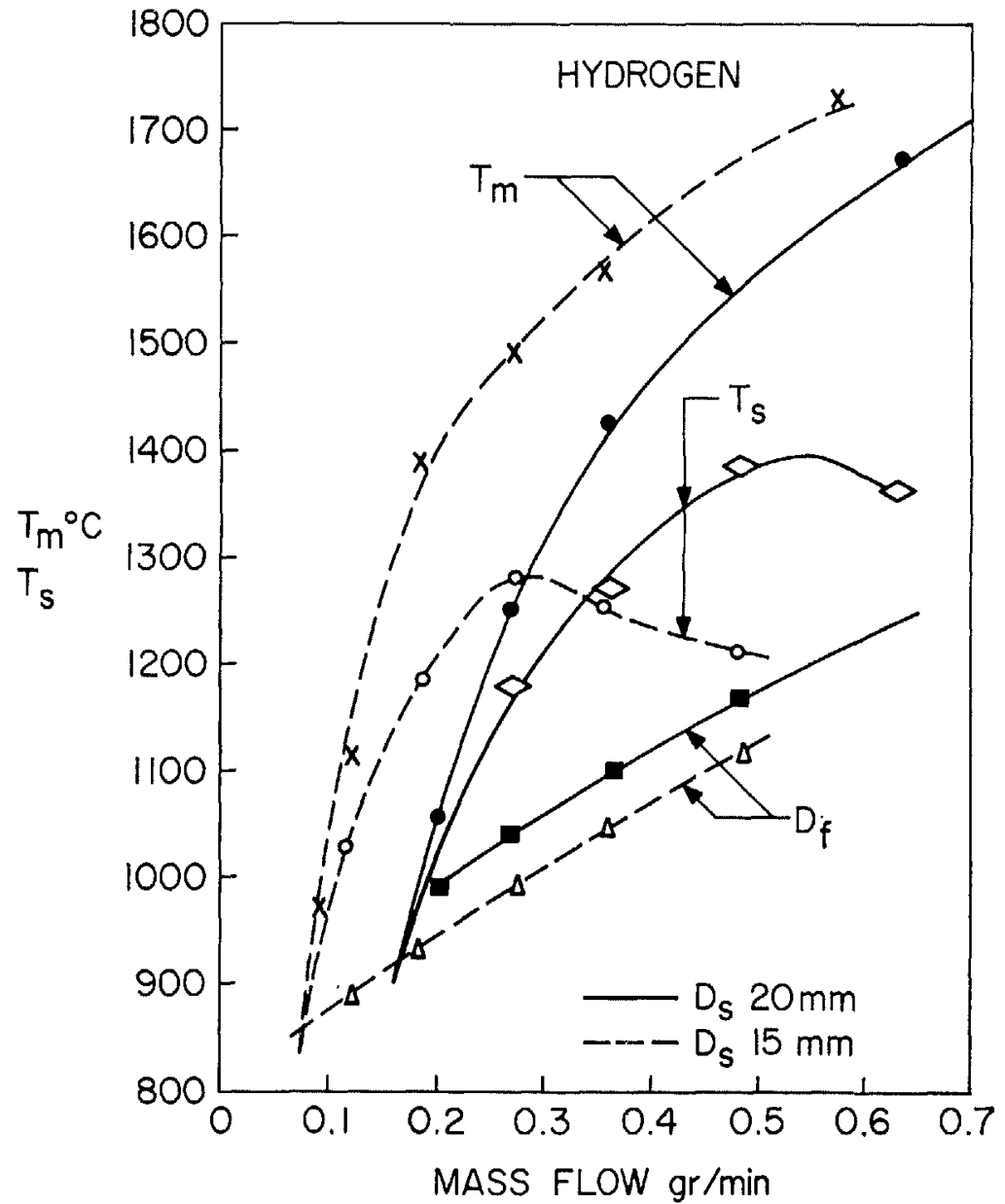

FIG. 5. Hydrogen-air diffusion flame. Experimental results. 
and experimental results or else they might be produced by a free convection effect.

Finally, flame diameters increased with mass flow in a form similar to that obtained theoretically, but depending on the sphere radius. This result could be expected because the influence of free convection on such a variable is particularly important.

\section{Nomenclature}

$\bar{c}_{p}$

Average value of the specific heats for the mixture

D Diameter

$e_{s} \quad$ Sphere emissivity

$\dot{m} \quad$ Mass flow injected through the sphere

$M_{j} \quad$ Molecular weight

$M$ Dimensionless mass flow

qr Heat of reaction

$r \quad$ Radius

$R \quad$ Dimensionless radius

$T \quad$ Absolute temperature

$w_{i} \quad$ Reaction rate

$Y_{i} \quad$ Mass fraction

$\epsilon_{i} \quad$ Ratio of flux of mass of species $i$ to total mass flow

$\bar{\lambda} \quad$ Average value of thermal conductivities for the mixture

$\mathfrak{L}_{i j} \quad$ Lewis-Semenov number

$\theta \quad\left(\vec{c}_{p} / q_{r}\right) T+\xi$, dimensionless temperature

$\xi \quad \frac{1}{q_{r}}\left[\frac{4 \pi r_{s}{ }^{2}}{\dot{m}} \sigma\left(T_{s}{ }^{4}-T_{\infty}{ }^{4}\right) e_{s}-\bar{c}_{p} T_{0}\right]$, dimensionless parameter

$\eta \quad$ Dimensionless ratio of the activation energy to the heat of reaction

$\sigma \quad$ Stefan Boltzmann constant

Subscripts

$i, j \quad$ Reactant species

1 Gaseous reactant from the porous sphere
Gaseous reactant surrounding the sphere Reaction products

Flame front

On the sphere surface

At infinity

Maximum value of temperature

Initial conditions of the gas injected through the sphere

\section{ACKNOWLEDGMENT}

The research reported in this document has been sponsored in part by the Office of Scientific Research, OAR through the European Office, Aerospace Research, United States Air Force.

\section{REFERENCES}

1. Sánchez Tarifa, C. and Ptregz del Notario, P.: Instituto Nacional de Técnica Aeronáutica. AFOSR TN 58-1038, 1958.

2. Sánchez Tarifa, C., Pírez del Notario, P., and García Moreno, F.: Eighth Symposium (International) on Combustion. Williams and Wilkins, 1962.

3. Sánchez Tarifa, C., Prerez del Notario, P. and García Moreno, F.: Instituto Nacional de Técnica Aeronáutica. AFOSR TN 62-25, 1961.

4. Sánchez Tarifa, C. and Ṕrezz del Notario, P.: Instituto Nacional de Técnica Aeronáutica. AFOSR TN 59-975, 1960.

5. Spalding, D. B.: Combustion \& Flame, 4, $51(1960)$

6. Spalding, D. B. and Jatn, V. R.: Combustion \& Flame, 5, 11 (1961).

7. Spalding, D. B., Jain, V. R., and Lamain, M. D.: Combustion \& Flame, 5, 19 (1961).

8. Wrstenberg, A. A. and Favin, S.: Combustion \& Flame, 4, 161 (1960).

9. Ṕ́rez del Notario, P. and Sánchez Tarifa, C.: Instituto Nacional de Técnica Aeronáutica. AFOSR Grant AF-EOAR 62-90, 1962.

\section{Discussion}

Dr. D. E. Rosner (AeroChem Research Laboratories): For the same reactant mass flow rate three distinct steady state solutions have been obtained. According to Professor Tarifa's comment, however, only one has any physical significance. In the theory of nonadiabatic reactors three distinct steady state solutions are frequently found, of which the two extreme ones represent systems which are statically stable (physically attainable) and the intermediate solution represents a system which is statically unstable (and therefore physically unattainable). In these cases the stable solution which is realized in practice depends upon the direction or method of attainment, and hysteresis effects are observed. Is it not possible that a similar situation exists for the laminar spherical flame system treated here? Could you elucidate the grounds on which you rule out the physical existence of two of the three steady state solutions?

Prof. C. S. Tarifa (National Institute of Technological Aeronautics, Madrid): I do agree with Dr. Rosner's comment regarding the stability of the intermediate solution. We studied such stability for 
the case of the premixed spherical flame of a monopropellant droplet and we found that the flame was unstablc.

Regarding the third solution, it corresponds to a theoretical combustion taking place at very low temperature (practically at ambient temperature) and through an extremely wide reaction zone. Flames of such type do not have physical existence. They have a mathematical existence due to the Arrhenius law which gives reaction rates different from zero for any value of the temperature.
Dr. R. B. Rosenberg (Insitute of Gas T'echnology): The question of the existence of a maximum temperature in the flame holder has been raised. We have observed the same phenomenon in the study of a circular, porous plate flame holder. We measured temperatures both by use of thermocouples and the sodium line reversal technique. This temperature maximum apparently is indicative of the mechanism of flame stabilization on this type of flame holder. 